CASO CLÍNICO

\title{
Síndrome de Wooblers en un caballo
}

\section{Wooblers syndrome in a horse}

\author{
José Cardona Á, ${ }^{1 *}$ M.Sc, César Betancur $\mathrm{H}^{1}{ }^{1}$ M.Sc, Jaime Alvarez $\mathrm{P}^{1}{ }^{1}$ M.Sc. \\ ${ }^{1}$ Universidad de Córdoba. Facultad de Medicina Veterinaria y Zootecnia. Departamento de Ciencias \\ Pecuarias. Montería, Colombia. *Correspondencia: cardonalvarez@hotmail.com
}

Recibido: Abril de 2010; Aceptado: Agosto de 2012.

\section{RESUMEN}

Se describe un caso de síndrome de Wooblers en un caballo, macho entero, de 14 años de edad, procedente del municipio de Cereté (Córdoba, Colombia). Esta patología es también conocida en equinos como mielopatía estenótica vertebral cervical (MEVC), la cual es una enfermedad del desarrollo caracterizada por estenosis del canal vertebral cervical resultando en compresión intermitente o continua de la médula espinal. A este paciente se le detectaron signos de incoordinación con tropiezos, ataxia simétrica, paresia o debilidad, espasticidad y pérdida de la propiocepción, principalmente en los miembros posteriores, por lo que se le realizó radiología cervical detectando estenosis de canal medular a nivel de la vértebra cervical C4. Estos hallazgos obedecen principalmente al síndrome de Wooblers.

Palabras clave: Ataxia, equino, Mielopatía (Fuente:CAB).

\begin{abstract}
A case of Wooblers syndrome is described in a complete male 14-year-old horse from the town of Cereté (Cordoba, Colombia). This condition is also known as equine cervical vertebral stenotic myelopathy (CVSM), which is a disease characterized by developmental cervical spinal canal stenosis resulting in intermittent or continuous compression of the spinal cord. This patient showed to have signs of incoordination which included stumbling, symmetrical ataxia, paresis or weakness, spasticity and loss of propioception, particularly in the hindquarters, so that radiology was performed to detect cervical spinal canal stenosis at the level of the cervical vertebrae C4. These findings are related as Wooblers syndrome.
\end{abstract}

Key words: Ataxia, equine, mielopaty (Source:CAB). 


\section{INTRODUCCIÓN}

El síndrome de Wooblers, mielopatía estenótica vertebral cervical (MEVC) o síndrome de incoordinación equina, es una enfermedad que se caracteriza por ataxia y debilidad, causada por el estrechamiento del canal vertebral cervical con o sin malformación de las vértebras cervicales, resultando en la compresión y/o trauma repetitivo de la médula espinal cervical, se considera una enfermedad multifactorial y es la principal causa de ataxia no infecciosa de la médula espinal en el caballo (1). Los caballos afectados con el síndrome de Wooblers generalmente son llevados a consulta debido a la debilidad y a la falta de coordinación, en edades comprendidas entre los 6 meses y los 4 años, aunque el problema puede ocurrir a cualquier edad (2), los machos enteros parecen ser los más comúnmente afectados con MEVC que las hembras; la razón para esta prevalencia no es clara, no obstante, esto puede ser el resultado de la influencia de la testosterona en el crecimiento o en el desarrollo de los osteocondrocitos (3).

Aunque no existe evidencia de que esta patología sea directamente heredable por patrones mendelianos recesivos, no se descarta el origen hereditario. Todas las razas pueden ser afectadas, presentándose con más frecuencia en caballos Pura Sangre Inglés y Cuarto de Milla, razas ligeras y de tiro; sin embargo, las razas ligeras y de tiro parecen ser las más predisponentes por su mayor exigencia para obtener productos de gran talla en el menor tiempo posible (4).

La causa principal de los signos clínicos es la estenosis; la cual puede ser el resultado de una malformación genética o un sobre crecimiento de los procesos articulares de las vértebras cervicales asociados con un rápido crecimiento o con desequilibrios nutricionales (5). Estos factores generan trastornos del desarrollo en los sistemas muscular y esquelético, trayendo como consecuencia problemas neurológicos por compresión del cordón espinal en diversos niveles de las vértebras cervicales $(2,6)$.

\section{CASO CLÍNICO}

En el presente reporte se describe un caso de MEVC en un equino mestizo criollo por cuarto de milla, macho entero de 14 años de edad, $500 \mathrm{~kg}$ de peso aproximadamente, dedicado a labores de trabajo (cochero), procedente del municipio de Cereté (Córdoba, Colombia), fue atendido por el servicio clínico ambulatorio de grandes animales de la Facultad de Medicina Veterinaria y Zootecnia de la Universidad de Córdoba, el cual presentó signos de incoordinación con tropiezos, ataxia simétrica, paresia o debilidad, espasticidad y pérdida de la propiocepción, principalmente en los miembros posteriores. Se le realizó exámen clínico general y neurológico especializado, consistente en evaluación de la cabeza, cuerpo entero, analizando postura y finalmente andadura.

La ataxia se evaluó neurológicamente, acentuándose más al efectuar círculos, elevar la cabeza o taparle los ojos y evadir obstáculos (Figura 1); al caminar, la debilidad se manifestó por tropiezos con arrastre de la punta del casco; al descansar se observó alteración de la propiocepción manifestada por una postura anormal (Figura 2). La espasticidad se evidenció por disminución de la flexión de la articulación de la rodilla al caminar. Cuando se le instó a moverse atrás (reculeo) el paciente abrió su base de apoyo e inclinó su cuerpo hacia atrás antes de mover sus miembros (Figura 3 ).

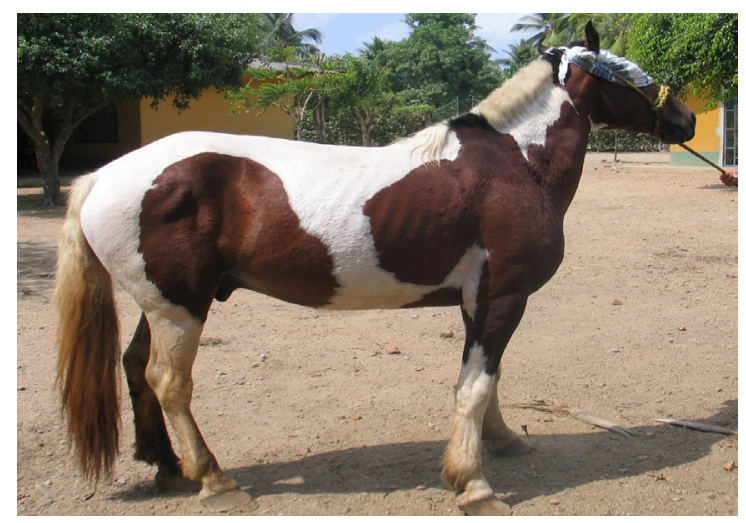

Figura 1. Evaluación neurológica de la ataxia, tapándole los ojos, nótese la renuencia a dar el paso, indicando acentuación de la ataxia.

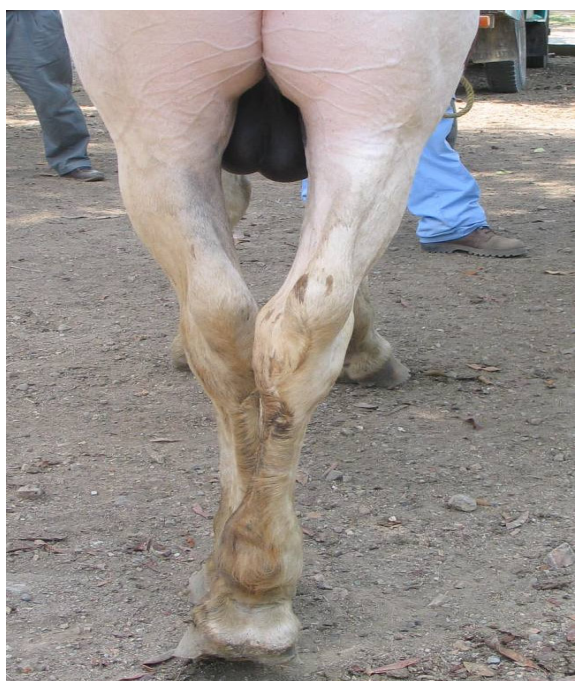

Figura 2. Posición anormal, después de realizar pruebas de propiocepción consciente, dejó el miembro en la posición colocada, debiendo ubicarlo nuevamente en su posición de apoyo. 


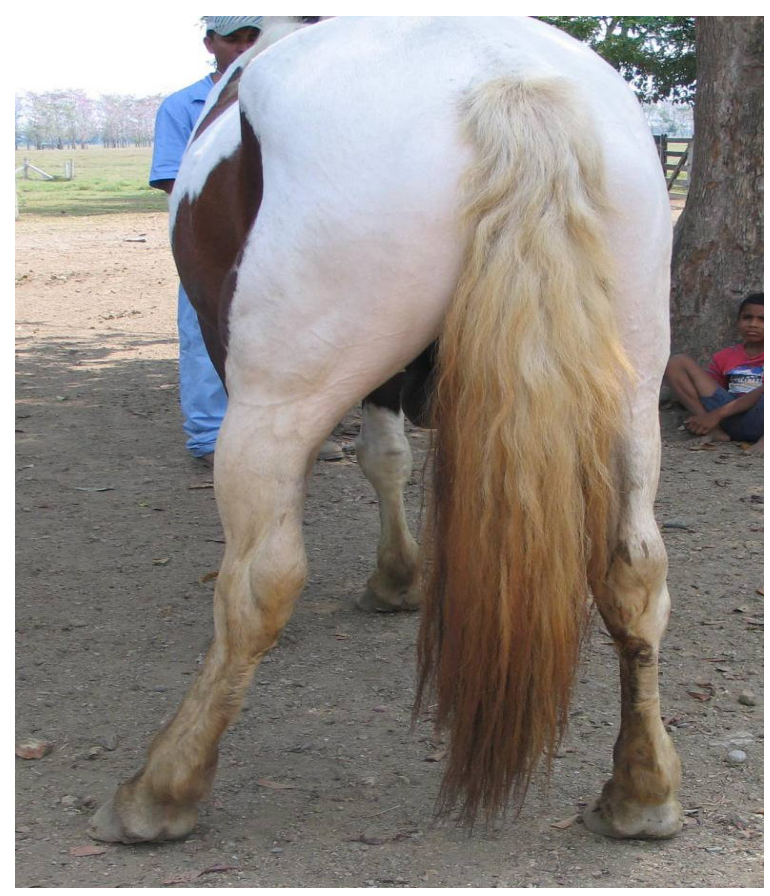

Figura 3. Movimiento hacia atrás (reculeo), el paciente abrió su base de apoyo e inclinó su cuerpo hacia atrás antes de mover sus miembros.

Hay dos manifestaciones clínicas de la enfermedad: La tipo 1, que tiende a ocurrir en animales jóvenes, desde el destete hasta los 2 años de edad, en este tipo se presenta malformación con estenosis del canal vertebral. Es una condición dinámica que ocurre de manera intermitente cuando el animal flexiona el cuello, más frecuentemente afecta las vértebras C3 - C4 y C4 - C5. Un trauma externo puede ser el factor que precipita inicialmente el síndrome clínico (7). La compresión de la médula espinal a nivel cervical en zonas donde las vertebras se encuentran inestables o malformadas, se deben principalmente a anormalidades del desarrollo (8).

La tipo 2 tiende a ser más frecuente en pacientes viejos con severa osteoartritis, la compresión es constante y es más común en la parte más caudal (C5 - C6, C6 - C7) (7). Estos caballos generalmente tienen una enfermedad vertebral cervical traumática adquirida (9).

Se recomendó un estudio radiográfico del cordón espinal cervical para determinar la probabilidad de MEVC, la cual fue realizada en la clínica de la Facultad de Medicina Veterinaria y Zootecnia de la Universidad de Córdoba, con el fin de valorar una posible malformación vertebral o la estrechez del canal vertebral se hicieron proyecciones laterales de cuello en el animal de pié.
Se detectó radiográficamente estenosis de canal medular a nivel de la vértebra cervical C4 (Figura 4), así como disminución del diámetro sagital vertebral (A/B) menor que 50\% (Figura 5).

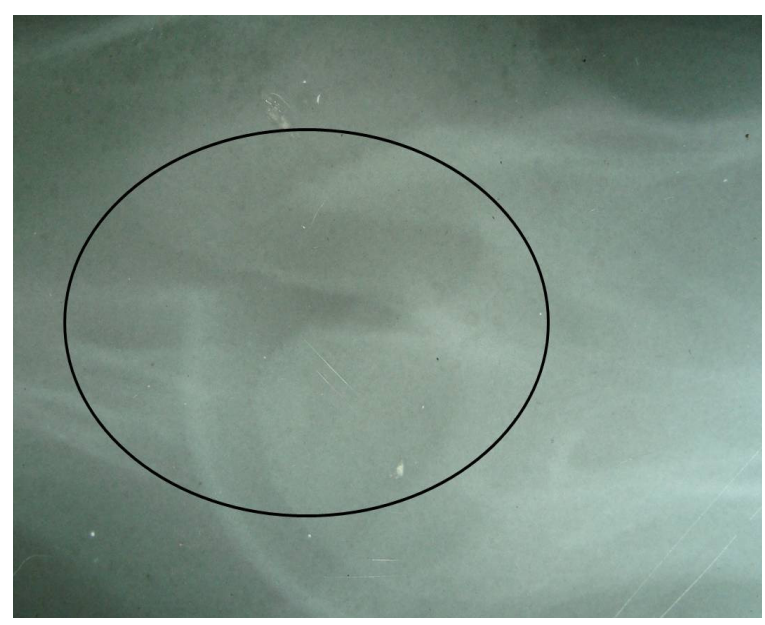

Figura 4. Estenosis del canal medular a nivel de la vértebra cervical C4.

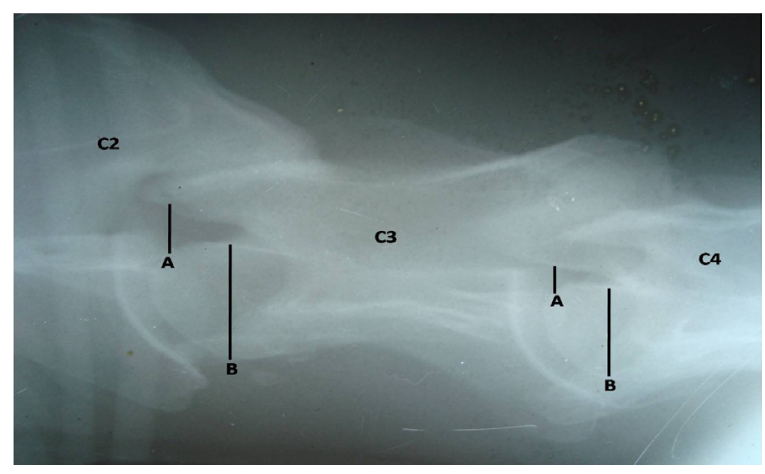

Figura 5. Disminución del diámetro sagital vertebral, menor que $50 \%(A / B)$ en $C 4$.

El diagnóstico presuntivo se fundamenta sobre la base de la historia clínica, del exámen clínico neurológico, la localización neuroanatómica y el exámen radiográfico lateral de las vertebras cervicales, obtenidas en el caballo de pie. La principal característica de malformación ósea de las vértebras cervicales en caballos con MEVC son "destellos" de las epífisis caudales en el cuerpo de la vertebra, osificación anormal del proceso articular, mala alineación entre las vértebras adyacentes, extensión de la lamina dorsal y la enfermedad articular degenerativa en el proceso articular de las articulaciones, caracterizada por osteocondrosis y/o artrosis del proceso articular, siendo la lesión más comúnmente identificada en las radiografías cervicales vertebrales en los caballos afectados con MEVC $(1,10)$. 
Es importante la difusión de este caso clínico en la literatura médica veterinaria dado que puede presentarse de 2 a $4 \%$ en los animales con mal manejo nutricional o físico, como lo reporta Moore et al (3) y que afecta a un $2 \%$ de caballos pura sangre (1).

\section{DISCUSIÓN}

Basados en los datos anamnésicos e historia clínica, la exploración física, el exámen neurológico y los hallazgos radiológicos, el diagnóstico final de este paciente fue MECV o síndrome de Wooblers.

En el examen radiográfico se observó estenosis del canal medular a nivel de la $4 a$ vértebra cervical que podría ser compatible con MECV, efectivamente se comprobó un canal vertebral estrecho a nivel de C4, demostrado por una disminución en el diámetro sagital mínimo (DSM), debido a que la interpretación de muchos cambios radiológicos es subjetiva, es necesario el uso de la relación sagital, ya que proporciona objetividad. Esto se calcula dividiendo el DSM en el aspecto craneal de la vértebra entre el diámetro del cuerpo vertebral en su parte más ancha y craneal; éste método elimina subjetividad y corrige por magnificación, tiene una sensibilidad y especificidad de $89 \%$ entre C4 y C7. Una relación sagital normal debe ser más del $50 \%$; si esta relación es menor que 50\%, es recomendado realizar un mielograma para confirmar la compresión medular. Si la relación es mayor que $50 \%$ el mielograma debe evitarse o posponerse, por lo que se deben considerar otros diferenciales. En este caso la relación fue menor que el 50\%, confirmándose ECVM, por lo que los resultados arrojados por el exámen radiológico, son compatibles con los reportados por Moore et al (3), Van Biervliet et al (11), y Rush y Grady (12).

El diagnóstico de este caso, se orientó hacia una ECVM del tipo II, por las características del paciente y por ser un equino geriátrico;sin embargo en la radiografía se observó estenosis del canal vertebral a nivel de C4, contrastando con lo informado por Mayhew I (7), quien indica que la ECVM tipo II es más frecuente en las vértebras C5 - C6 y C6 - C7, aunque el paciente pudo llevar mucho tiempo padeciendo el proceso. En equinos, la etiología de MECV es multicausal, se cree en la etiología de origen genético, además se han considerado causas ambientales, no se estableció la genealogía del paciente, lo cual podría haber servido para identificar factores hereditarios, propuesto por Stewart et al (13).

Es importante tener como ayuda diagnóstica a la radiología, ya que de esta manera se puede determinar la estenosis del canal o los cambios degenerativos articulares, así como la alineación vertebral y asociarlos contundentemente a los signos clínicos neurológicos.

En conclusión, los equinos manejados en explotaciones ganaderas de alta producción, están predispuestos a sufrir de enfermedades metabólicas y degenerativas, debido a la falta de un adecuado programa de alimentación estratégico para equinos de trabajo, siendo este un factor determinante en la presentación de este tipo de casos, ya que se ha reportado mejoría en caballos con esta enfermedad, al ser aplicados tratamientos nutricionales y de manejo conservatorio (14).

Las terapias pueden ser conservadoras o sofisticadas, siendo ambas costosas y con resultados inconsistentes, por lo que es conveniente establecer en los criaderos programas de alimentación y manejo adecuados con la finalidad de disminuir la incidencia de estos casos. También se postula la corrección quirúrgica, como tratamiento más reconocido de esta condición (15).

Se recomendó al propietario la realización de un tratamiento médico y conservatorio informándole además acerca de la gravedad del proceso y por lo incierto del pronóstico, se decidió practicar la eutanasia al paciente 


\section{REFERENCIAS}

1. Reed $\mathrm{S}$, Equine cervical vertebral myelopathy - surgical, medical, and dietary management of affected horses and outcome. Orlando, Florida: Proceeding of the North American Veterinary Conference; 2007.

2. Pujol B, Mathon D. Le Wobbler Syndrome chez le cheval. Spondylomyélopathie cervical ou «Mal de chien» Etude bibliographique Première partie. Rev Méd Vét 2003; 154(3):211-224.

3. Moore B, Reed S, Biller D. Assessment of vertebral canal diameter and bony malformations of the cervical part of the spine in horses with cervical stenotic myelopathy. Am J Vet Res 1994; 55:5-13.

4. Araujo L, Da Rosa J, Paiva L, Nunes M, Wayne C. Mielopatia cervical estenótica em equinos - estudo de 7 casos. Ci Anim Bras, Goiânia 2009; 10(3):990-996.

5. Stewart R, Reed S, Weisbrode S. Frequency and severity of osteochondrosis in horses with cervical stenotic myelopathy. Am J Vet Res $1991 ; 52: 873-879$.

6. Filippini S, Catalano L. Martuzzi F. Anomalie di sviluppo dell'apparato locomotore nel puledro in relazione alle tecniche d'allevamento. Ann Fac Medic Vet di Parma 2004; XXIV:247-256.

7. Mayhew I. The Healthy Spinal Cord. Proceedings AAEP 1999; 45(B):56-66.
8. Nout Y, Reed S. Cervical vertebral stenotic myelopathy. Equine Vet Edu 2003; 15:212-223.

9. Yovich J, Le Couteur R, Gould D. Chronic cervical compressive myelopathy in horses: clinical correlations with spinal cord alterations. Aust Vet J 1991; 68:326-334.

10. Levine J, Adam E, Mackay R. Confirmed and presumptive cervical vertebral compressive myelopathy in older horses: a retrospective study (1992-2004). J Vet Intern Med 2007; 21:812-819.

11. Van Biervliet J, Mayhew I, Delahunta A. Clinical Vertebral Compressive Myelopathy. Clin Tech Equine Pract 2006; 5:54-59.

12. Rush B, Grady J. Cervical Stenotic Myelopathy. Compendium Equine 2008; 430-437.

13. Stewart R, Reed S, Weisbrode S. Frequency and severity of osteochondrosis in horses with cervical stenotic myelopathy. Am J Vet Res 1991; 52:873-879.

14. Vázquez-Rangel F. Síndrome de incoordinación motora en caballos (Wobbler). Rev Sanid Milit Mex 2006; 60(4):278-282

15. Walmsley J. Surgical treatment of cervical spinal cord compression in horses. Equine Vet Edu 2005; 17:39-43. 